\title{
THE QATAR NATIONAL HISTORIC ENVIRONMENT RECORD: A PLATFORM FOR THE DEVELOPMENT OF A FULLY-INTEGRATED CULTURAL HERITAGE MANAGEMENT APPLICATION
}

\author{
R. T. H. Cuttler ${ }^{a}, *, T . W . W$. Tonner $^{b}$, F. A. Al-Naimi ${ }^{c}$, L. M. Dingwall $^{a}$ and N. Al-Hemaidi ${ }^{c}$ \\ ${ }^{a}$ Institute of Archaeology and Antiquity, University of Birmingham, Edgbaston, Birmingham, UK - \\ r.cuttler@bham.ac.uk,1md104@bham.ac.uk \\ b coaptics ltd., 2 New Hospital Villas, Hospital Road, Talgarth, LD3 0DU, UK - tobi.tonner@ coaptics.co.uk \\ c Department of Antiquities, Qatar Museums Authority, P.O. Box 2777, Doha, Qatar - falnaimi@qma.com.qa
}

KEY WORDS: Cultural Heritage, Geospatial Database, GIS, Management, Application, Software, Web based

\begin{abstract}
:
The development of the Qatar National Historic Environment Record (QNHER) by the Qatar Museums Authority and the University of Birmingham in 2008 was based on a customised, bilingual Access database and ArcGIS. While both platforms are stable and well supported, neither was designed for the documentation and retrieval of cultural heritage data. As a result it was decided to develop a custom application using Open Source code. The core module of this application is now completed and is orientated towards the storage and retrieval of geospatial heritage data for the curation of heritage assets. Based on MIDAS Heritage data standards and regionally relevant thesauri, it is a truly bilingual system. Significant attention has been paid to the user interface, which is userfriendly and intuitive. Based on a suite of web services and accessed through a web browser, the system makes full use of internet resources such as Google Maps and Bing Maps. The application avoids long term vendor 'tie-ins', and as a fully integrated data management system, is now an important tool for both cultural resource managers and heritage researchers in Qatar.
\end{abstract}

\section{INTRODUCTION}

\subsection{Rationale}

When we record cultural heritage, a significant amount of effort is often invested in individual sites, buildings or objects, capturing in great detail their character, form and condition. While such highly detailed records are invaluable, in reality the collection of data is simply an initial stage of management, maintenance and conservation. How this data is assembled, curated and accessed is fundamental to the effective management of cultural resources.

A prerequisite to safeguarding heritage assets in each country is a system that informs managers about the entirety of the cultural heritage resource, from regionally important ephemeral archaeological sites (including those known to have been destroyed), to internationally important world heritage sites. Such a system should not be mired in detail, but should be an intuitive index that aids discovery, management, research and education.

To realise this goal, Qatar developed their first National Historic Environment Record (QNHER) using existing software packages. As a robust system for cultural resource management the QNHER provided a platform for the subsequent development of software designed specifically for the purposes of cultural heritage management.

\subsection{Background}

Between 2008 and 2009, the Qatar Museums Authority (QMA) and the Institute of Archaeology and Antiquity at Birmingham University collaborated on the analysis of terrestrial and marine remotely sensed data. The aim was to use these data to identify and document archaeological sites and palaeoenvironmental remains within Qatar. It soon became clear that the pre-existing national inventory was inadequate for recording and retrieving what was anticipated to be a large dataset.

As a consequence the first version of the QNHER (Beardmore et al. 2010) was built in close partnership with the Centre for GIS, the official mapping agency and hub for spatial information in Qatar. The challenge was to develop a cultural resource management tool for both monuments on land and the submerged cultural heritage, while integrating new data from remote sensing with existing records. Since becoming 'live' in 2009 this has been an invaluable resource for cultural heritage research, management and mitigation (Beardmore et al. 2010) and currently holds almost 6,000 cultural heritage records.

\subsection{Existing system review}

Prior to developing the first QNHER version several heritage management systems used in the UK were considered, but found to be unsuitable. Those developed in the mid 1990's are characterised by expensive per-seat licencing, dependability on other commercial software packages and little scope for major customisations (e.g. "HBSMR" by exeGesIS). In addition, a multilingual software package (in this case Arabic and English) was an essential requirement, but unfortunately not available. The only existing application using a bilingual platform was far too complex and future system support unclear ("Archwilio" used by the Welsh Archaeological Trusts).

A common issue was that existing systems were dominated by complex user interfaces which make for a steep learning curve. A custom system offered the opportunity for bilingual thesauri based on regional data standards, terminologies and work-flow, and as a result development of this system subsequently commenced in 2008 (Beardmore et al. 2010). A detailed

\footnotetext{
* Corresponding author.
} 
overview and comparison of existing systems is beyond the scope of this article.

\subsection{System development}

Guided by the MIDAS Heritage data standard (v1 2007, now updated to v1.1, English Heritage 2012), a Microsoft Access database was designed which allowed the recording of archaeological sites, activities/events and bibliographic references. The database was linked to ESRI ArcGIS via crossreferencing of unique record identifiers to display the spatial location of archaeological sites and activities. Emphasis was given to a simple, bilingual user interface, comprehensive site reports and data flow with other government ministries (Beardmore et al. 2010).

\subsection{Rationale for QNHER 2.0}

As the database was populated the QNHER formed an invaluable cultural heritage management tool, particularly for the provision of a mitigation response in the face of threats posed to heritage by infrastructure development. However, it was also clear that the system had inherent limitations, and further development would be necessary to meet growing demands and ensure its future success.

Three major factors drove the development of QNHER 2.0:

1. Accessibility, availability and sharing

2. Seamless integration of information

3. Long term feasibility and sustainability

Accessibility, availability and sharing: A fundamental principle of QNHER 2.0 is data accessibility for all stakeholders. Sharing this resource ensures that heritage forms part of an informed decision making process, and is of benefit to all through either contributing or utilising information.

The seamless integration of information: The QNHER recorded textual, spatial and pictorial information using a combination of software platforms. A single system with an intuitive interface, able to manage different kinds of data in an integrated way, was considered more appropriate. Successful cultural heritage management relies on providing a visible context to all available information.

Long term feasibility: While the argument for proprietary applications is their sustainability through license funding, Open Source alternatives offer greater flexibility and avoid long-term 'vendor tie-ins'. Open Source also offers the potential for further modules that are not based around a vendor's core application. Furthermore a custom system offered the opportunity to separate user interface concerns from the underlying server technology, making it easier for other applications to interface with the system. Finally, as the intellectual property rights of the software belong to the QMA, it can proactively manage how the system is used and also how it may be shared with other organisations.

\section{IMPLEMENTATION}

\subsection{QNHER 2.0}

The QNHER 2.0 allows for the recording and retrieval of a comprehensive and fully-integrated range of heritage information, including information relating to archaeological and historic sites, buildings and other structures, heritage complexes and landscapes, designated legal statuses, excavations, surveys and other fieldwork, site monitoring and significance assessments, geology, topography and landuse, artefacts and ecofacts, and textual and photographic references. The interlinking of all this information within a single system offers greater potential for interpretation and analysis of this resource.

\subsection{Data standards and entities}

In line with the first version, QNHER 2.0 is based on MIDAS Heritage data standards (English Heritage 2012). Notably some aspects have been adapted for use in Qatar, including the terminology used for the QNHER entities. Table 1 summarises these and their relationship with MIDAS Heritage Information Groups.

\begin{tabular}{|c|c|}
\hline QNHER Entity & $\begin{array}{l}\text { Related MIDAS Heritage } \\
\text { Information Groups }\end{array}$ \\
\hline $\begin{array}{l}\text { Heritage Area } \\
\text { (Sites) }\end{array}$ & $\begin{array}{l}\text { Monument } \\
\text { Artefact and Ecofact } \\
\text { Date and Period } \\
\text { Map Depiction }\end{array}$ \\
\hline $\begin{array}{l}\text { Heritage Activity } \\
\text { (Events) }\end{array}$ & $\begin{array}{l}\text { Investigative Activity } \\
\text { Heritage Asset Management } \\
\text { Activity } \\
\text { Research and Analysis } \\
\text { Historical Event } \\
\text { Date and Period } \\
\text { Map Depiction }\end{array}$ \\
\hline $\begin{array}{l}\text { Management } \\
\text { Area }\end{array}$ & $\begin{array}{l}\text { Designation and Protection } \\
\text { Date and Period } \\
\text { Map Depiction }\end{array}$ \\
\hline Organisation & Actor and Role \\
\hline Person & Actor and Role \\
\hline Resource & $\begin{array}{l}\text { Archive and Bibliography } \\
\text { Management Activity } \\
\text { Documentation } \\
\text { Map Depiction }\end{array}$ \\
\hline Image & $\begin{array}{l}\text { Archive and Bibliography } \\
\text { Management Activity } \\
\text { Documentation } \\
\text { Map Depiction }\end{array}$ \\
\hline
\end{tabular}

The QNHER entities are not independent from each other, but in most cases a complete record only emerges when information from various entities are interlinked.

For example a Heritage Area (Site) will always be related to the Heritage Activity (Event) which first made us aware of the Heritage Area. The Heritage Activity in turn will in most cases be documented in either a Resource and/or Image, and therefore would be linked to these entities.

Apart from links between different entities, Heritage Areas and Heritage Activities can have internal links with records of the same entity to indicate relationships between records. These relationships can be either of a peer-to-peer or parent-child nature.

Where appropriate, values for data fields (also called 'units of information' in MIDAS Heritage) are controlled via terminology lists which were compiled specifically for use in Qatar. This includes, for example, lists of valid terms for Heritage Area types and time periods. 


\subsection{Technical standards}

For the QNHER 2.0 a stateless web service based on Representational State Transfer (REST) principles was implemented. These were first defined by Fielding (Fielding 2000) and have now become a common and efficient way of exposing resources via the Internet. Crucially RESTful web services make use of the core architecture of the Hypertext Transfer Protocol (HTTP) by using the methods GET, PUT, POST and DELETE to interact with resources which are made available via Uniform Resource Identifiers (URIs).

The QNHER 2.0 mapping services use Open Geospatial Consortium (OGC) compliant protocols (WMS and WFS) which have become de facto standards for submitting spatial information via the Internet in the geospatial industry.

An ACID (Atomicity, Consistency, Isolation, Durability) compliant relational spatial database was chosen as the database platform for the QNHER 2.0 to guarantee that data and associated transactions are processed reliably.

The QNHER 2.0 User Interface was designed around the Model-View-Controller (MVC) paradigm which separates data storage from visualisation and interaction aspects. This has become an important basis for the development and maintenance of complex, Rich Internet Applications (RIA).

\subsection{Architecture}

One of the key requirements was to separate client and server concerns. This was achieved through the implementation of a range of stateless web services which could be used by any interface or system that required access to the QNHER 2.0. The solution comprises three main layers:

1. Data storage: Textual and spatial information is stored in a database and binary information in the file system.

2. Web Application Programming Interfaces (APIs): These expose the data storage layer as a set of open and generic services.

3. User Interface (UI): Retrieves and sends data via Web APIs and exposes all available functionality to the user.

All parts of the system are based on Open Source components and applications, giving full control over all parts of the system and avoiding vendor and functionality lock-in.

The Web APIs are exposed to the Internet via a proxy server and all traffic between the client and the server is encrypted using the HTTPS protocol (Figure 1). Access to all layers of the system is controlled via user authentication. Automatic data backups ensure data is secured for the long term.

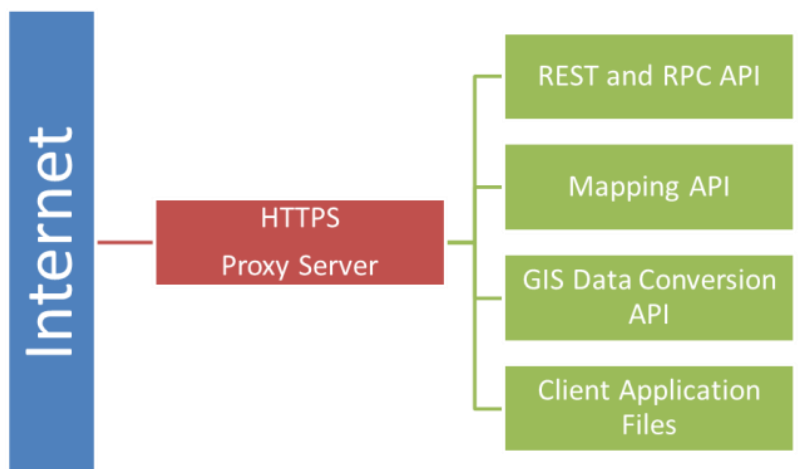

Figure 1. General architecture of the QNHER 2.0

\subsection{Development frameworks and software}

An important factor in the choice of development frameworks and software was the ability to deploy the system on both Linux and Windows operating systems and therefore making the QNHER 2.0 less dependent on a specific software environment. The same applied to the client where we aimed to support desktop and mobile devices running various operating systems.

On the server: To store the textual and spatial information we chose the world's leading Open Source database PostgreSQL (http://www.postgresql.org) with the added spatial extension PostGIS (http://postgis.net). Node.js and associated Open Source software is used for most of the Web APIs (http://nodejs.org) with the main exception being the Mapping API which is powered by the popular Open Source software GeoServer which runs on Java (http://geoserver.org).

Image processing is carried out using the ImageMagick library (http://www.imagemagick.org), conversion of GIS files is done by ogr2ogr (http://www.gdal.org/ogr2ogr.html) and PDF printing is supported via the wkHTMLtoPDF library (https://code.google.com/p/wkhtmltopdf). All these external libraries are called from within Node.js and specific functionality is exposed as part of the suite of web services.

On the client: The QNHER 2.0 User Interface was built using HTML5 technologies which are now widely supported across many different web browsers, operating systems and devices.

The core of the user interface is powered by two JavaScript frameworks: OpenLayers as the mapping interface (http://openlayers.org) and the Dojo Toolkit for all other client functionality, including UI design, forms and communication with the APIs (http://dojotoolkit.org).

\subsection{REST and RPC API}

Although the details of the QNHER 2.0 REST API are beyond the scope of this article, some special features should be mentioned here. Beyond the basic functionality of creating, reading, updating and deleting information, the REST API is secured via user authentication, provides powerful querying capabilities and can perform batch processing, image manipulation and PDF printing.

Remote Procedure Calls (RPCs) are used to instantiate processes from the client on the server. This allows the user to perform tasks which cannot be easily carried out on the client, such as image manipulation and PDF printing.

User authentication: As the QNHER 2.0 REST API is available via the Internet, appropriate security measures have to be put in place to avoid unauthorised access. To access any of the resources, the client needs to authenticate the user first. This is achieved via an RPC on the REST API.

Querying: Queries allow clients to accurately retrieve relevant subsets of QNHER information. Supported query operations include sorting, selecting a subset of fields, retrieving distinct records, requesting a record range, counting the number of results, wildcard searches, regular expression searches, retrieving records with empty properties and more. All these query operations can be combined in a single GET request to narrow down a result set.

Batch processing: Instead of sending individual requests, the REST API supports a batch POST request which bundles any number of GET, PUT, POST and DELETE request into a single 
call. The advantage of this approach is that it reduces HTTP traffic and improves overall performance.

Image manipulation: Image manipulation is currently limited to resizing and extraction of EXIF metadata. The resizing functionality can be used to generate web-ready thumbnails and larger on-screen sizes of Images. The EXIF extraction reads the metadata stored in the Image and returns it to the client which in turn can use this information to populate the system (e.g. GPS location).

PDF printing: The QNHER 2.0 system can use the PDF printing RPC to generate reliably formatted outputs for reports and maps.

\subsection{Mapping API}

The Mapping API provides access to spatial information stored in the QNHER via a Web Map Service (WMS) and a Web Feature Service (WFS). The latter allows authenticated users to edit the spatial information from a WFS compatible client (e.g. the QNHER client or other GIS applications). An additional feature of the WFS is the ability to specify 'Shapefile' as an output format which enables external systems to download information in a standard GIS format. Other outputs (KML, GeoRSS, etc.) exist which allow data exchange with other external system such as Google Earth. User authentication is handled separately from the REST API via the GeoServer builtin mechanisms. A more detailed description of the Mapping API is beyond the scope of this paper.

\subsection{GIS Data Conversion API}

This API allows client to convert GIS file formats (Shapefile, MapInfo, AutoCAD, etc.) into GeoJSON for use in web applications. This can be useful when existing GIS data needs to be integrated into the QNHER dataset.

\section{USER INTERFACE}

\subsection{Design}

The foremost consideration when designing a User Interface is the end-user, their level of expertise and their understanding of the subject matter. The aim was to make the QNHER 2.0 client application self-explanatory for heritage management professionals and to provide simple-to-follow dialogues and wizards where appropriate. Common operations have been optimised for efficiency and attention paid to displaying regular feedback when operations have been successful or have failed. In the latter case details are displayed on how to proceed. Considerable attention was also given to facilitating information retrieval and output, a very important area of work that is often neglected in user interface designs in comparison to data entry tasks.

The User Interface is fully bilingual, currently supporting English and Arabic language. Translations are not limited to the application itself, but also cover all terminology lists and data fields. This enables true bilingual use of the system.

A major consideration for the User Interface layout was to maximise the use of available screen space. Many applications 'resolve' this issue by overlaying separate windows on top of each other with the result that the user is unable to display all relevant information in its context (e.g. a record details window may be displayed overlaying a map which shows the location of the record).

To avoid this the QNHER 2.0 User Interface splits into three areas: data lists on the left for browsing and searching records, a map in the upper right for interaction with spatial records and a record detail area in the lower right part of the screen for viewing and editing records (Figure 2). It is worth noting that this layout swaps to right-to-left when the language is switched to Arabic.

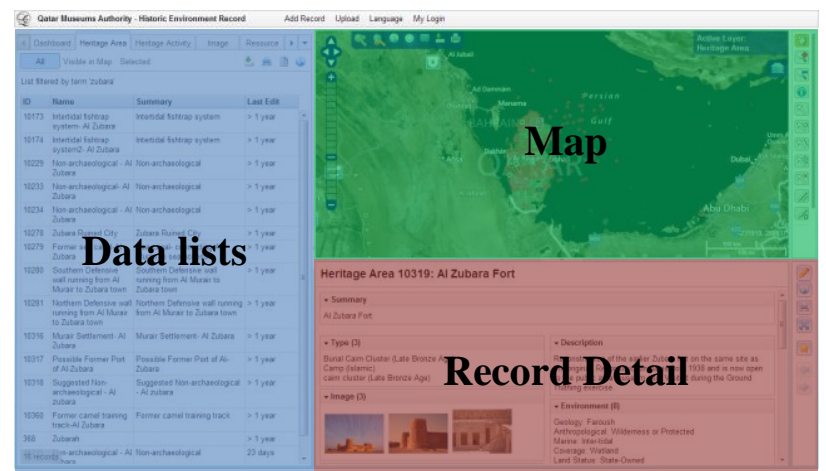

Figure 2. Layout of the QNHER 2.0 client application. The layout changes from right to left when switching to Arabic

The dividers between these areas can be moved to adjust the space to suit the relevant activity. The overall size of the layout is determined by the size of the screen used. A minimum screen resolution of $1024 \times 768 \mathrm{px}$ is recommended. The application was designed to support touch gestures which means that it can also be used on tablets and other touch-based interfaces.

\subsection{Data lists}

The data lists area is the central hub of all information stored in the QNHER. We've intentionally designed this to be similar to a spread sheet program as most users are familiar with this format (Figure 3).

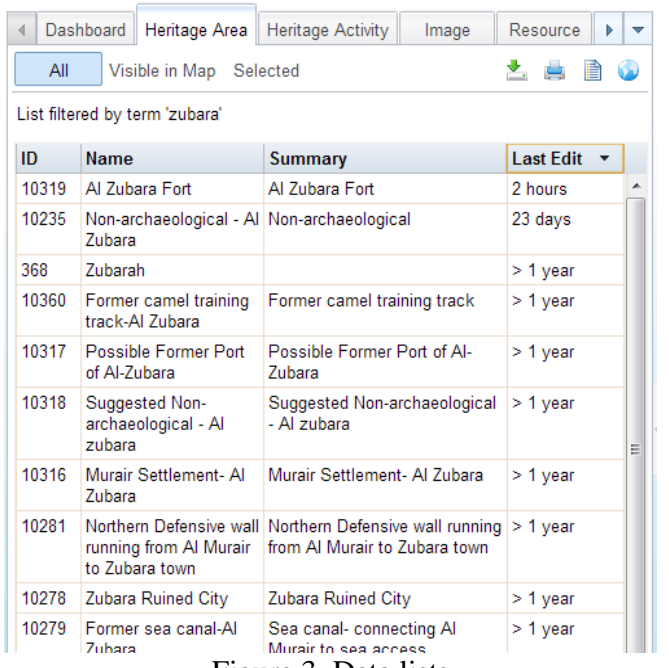

Figure 3. Data lists

Display modes: Each QNHER entity is displayed on its own 'sheet' and there are three available modes for viewing the data:

1. All: View and search all the information stored in the system for this entity

2. Visible in Map: Only show records that are currently visible in the map 
3. Selected: Only show records that the user has selected in the list and/or on the map

These three modes allow for very efficient interaction with the data depending on the relevant task. When a mode is selected the list displays summary information for the records. Columns can be sorted by clicking on the headers and lists of several thousand records can be scrolled without delay. Note also that each list contains a column 'Last Edited' which displays when the record was last modified. This is particularly useful when working with complex, highly interlinked records.

Tools: Several tools are available including the ability to download the records displayed in the list, print them in a PDF report, create a selection from the displayed records and view the record distribution in the map.

Search: Two types of searches can be performed: a quick search which is limited to keywords and a selection of commonly queried fields and an advanced search which allows for complex queries to be designed. The advanced search also supports compound queries, which can be combined with "and" / "or" statements and result in unlimited querying flexibility. The search facility also supports searching for "no value" and "any value", a task which is very important for data management, but is often difficult or absent in other systems.

Selection: Records can be selected in the list to create custom selections. This functionality is closely integrated with the map so that any records selected/highlighted in the list are also selected/highlighted in the map and vice versa.

\subsection{Dashboard}

The dashboard is part of the data list area and presents the user with information relating to their personal use of the system. It includes a list of recently modified records which is particularly useful to quickly access and interlink currently relevant records. There is also a bookmark area which can be populated by the user with records that are of importance to the current tasks.

\subsection{Map}

The map area is vital component of the system and its layout was optimised to provide as much visible map area as possible (Figure 4). Similar to other mapping systems, various background mapping layers can be activated by the user. All the standard tools a user nowadays expects from an online map exist (zoom in/out, pan, identify, select, measure, zoom to coordinate, etc.). Beyond this special tools required for heritage management work have also been added:

Buffer selections: By digitising a line, point or polygon, the system can buffer this object and then select spatial objects that intersect with the buffer. This is especially useful for creating complex selections, e.g. for road or pipeline development schemes.

Spatial data export: The system can create Shapefiles containing either the records visible in the map, the current record selection or all records of an entity. This is useful for extracting specific spatial information so that it can be provided to external bodies.

Map printing: The system can print the current map view to $\mathrm{PDF}$ in various sizes (A4 - A0).
Contrast/Brightness adjustments: Background mapping can often be of low contrast and we have added a tool which allows the user to adjust this to help identify features on the ground which are otherwise not clearly visible.

Auto-save: Instead of relying on the user to save changes manually, the system performs this task automatically.

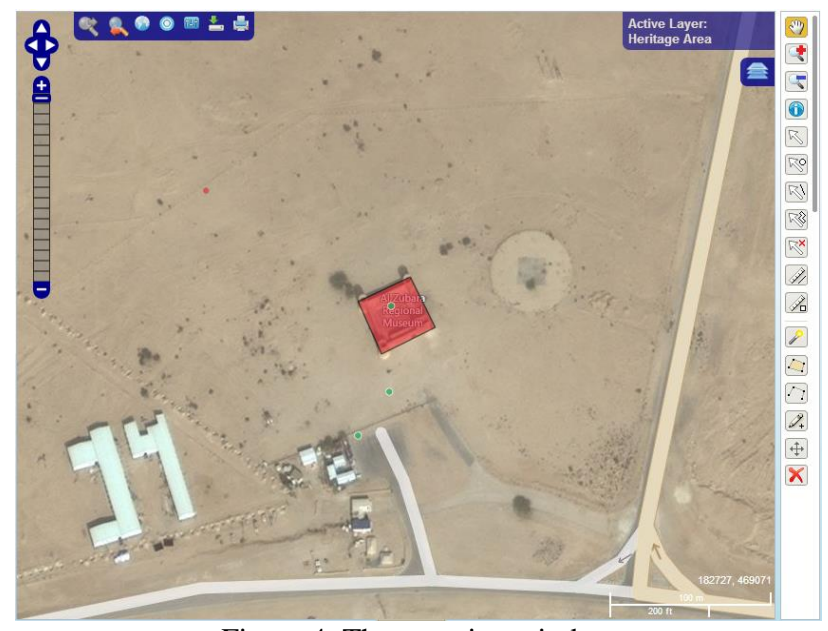

Figure 4. The mapping window

\subsection{Record detai}

The record detail area operates in two distinct modes: A browse mode for viewing information and navigating between records (Figure 5) and an editing mode for modifying the record. The latter is only available for users with editing permissions on the record. Various tools exist in this area, including toggling between browsing and editing modes, printing a PDF report of the record, viewing it on the map, bookmarking it and moving forwards/backwards in the record navigation history. Modifications are automatically saved when the user navigates away from a record and validation checks can be reviewed on manual save.

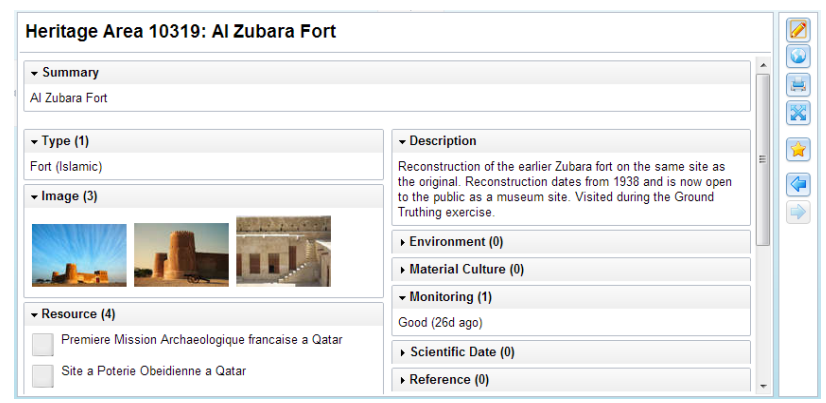

Figure 5. Record detail browser form

\subsection{Workflows}

Workflows are handled to ensure that users can easily perform common tasks while enforcing data quality and performing data validation. New records for example are created via a simple wizard that guides the user through several steps until all mandatory information has been entered.

One regular operation is the association between individual records, such as the link between a Heritage Area (Sites) and any Heritage Activities (Events). These associations are easily created by dragging records from the data lists and dropping them into the record detail area. This method also works for 
linking images and documents to records. Navigation between records can be performed by double-clicking on a related record. A history of records visited during a user session is kept in memory and 'forwards' and 'backwards' buttons can be used to navigate this history.

\subsection{Image and document management}

The QNHER 2.0 system treats images and documents as entities in their own right. This means that individual image and document records store detailed information about content, dates and authors and can be linked to many other records in the system without duplication. Simple upload wizards are provided to make adding these resources as efficient as possible.

\section{CONCLUSIONS}

It is no exaggeration to say that the QNHER 2.0 is a milestone in cultural resource management software development. It integrates many of the capabilities previously only available by sharing and analysing data across a combination of software platforms. This integration of information is seamless. Thesauri, data standards and importantly, the work-flow established during the development of the first version of the QNHER provided an ideal platform for the steady stream of everyday steps, tasks and interactions around which QNHER 2.0 could be designed and beta-tested.

However the vision behind QNHER 2.0 was not merely for data storage but to provide tools to query, analyse and easily retrieve data in an appropriate manner. The development of these tools benefited from an on-going dialogue between the system developers and end users from design stage to beta-testing. Perhaps even more fundamental was the fact that the software and data architecture was designed and developed by heritage data management specialists with extensive experience of cultural resource management. This provided a very necessary understanding of the needs and challenges faced by the end users.

The vision for QNHER 2.0 also included an intuitive User Interface that would make the system accessible to users with different levels of experience and expertise. The more complex an application (particularly where extensive training is required), the correspondingly fewer number of users. An intuitive system that is extensively used is considerably more desirable than a complex system that remains unused. The challenges for the development of future modules is to maintain this intuitive format, while at the same time developing greater functionality in specialist areas.

A multilingual user interface is also an essential requirement, as it encourages a wider range of users. Working in close liaison with Qatari heritage experts has ensured that the terminology, lists and data fields are appropriately translated. This has resulted in a system that is truly bilingual. However, translation will present an on-going challenge. Modifications in one part of the software will result in further changes to either the Arabic or English. While it is preferable for records to be entered in Arabic, the ongoing translation of records means that QNHER 2.0 is fully accessible to visiting teams and researchers.

As an intuitive, fully-integrated data management system QNHER 2.0 is now used internally by the QMA as the basis of the recording, research and conservation of cultural heritage in Qatar, but it could in future also have implications for heritage management in other regions. Clearly the diversity of cultural heritage, site types and chronology across not just other parts of the globe, but within the Arabian Peninsula, means that simply attempting to transplant a system is inappropriate. However, with the input of regional heritage managers, particularly with regard to language and thesauri, the system could be customised to address the needs of cultural resource managers in other countries. Internet resources and base mapping such as that provided by Google Maps and Bing Maps may play a significant role in this. In Qatar access to georeferenced base mapping is made available to government ministries through the Centre for GIS which also acts as a hub for exchanging spatial information, including heritage datasets. However, this is the exception rather than the rule. Most antiquities departments around the globe do not have country-wide, georeferenced base mapping. Access to internet resources has major cost-saving benefits for antiquities departments, while providing improved mapping and data visualisation. More importantly this offers the opportunity for cultural heritage management tools to be established with minimal outlay and training.

For any specialist software sustainability can be a significant and uncertain issue. The commitment of the Qatar Museums Authority to QNHER 2.0 provides the means to avoid many of the longer term issues associated with custom systems, such as maintenance and software updates. Plans for further public access, Underwater Cultural Heritage, buildings and tabletbased survey modules will also promote a sustainable system. QNHER 2.0 is not just a tool that offers improved public accessibility to heritage information, nor is it merely an educational tool. In the longer term the real value of this system lies in the pro-active management and protection of the heritage resource, from designation and curation to forward planning and future research

\section{REFERENCES}

Beardmore. R., Cuttler, R., Al-Naimi, F., Ramsey, E., Fitch, S., Kallweit, H. 2010. The Qatar National Historic Environment Record: a bespoke cultural resource management tool and the wider implications for heritage management within the region. Proceedings of the Seminar for Arabian Studies, 40, pp. 43-54.

English Heritage, 2012. MIDAS Heritage - The UK Historic Environment Data Standard, v1.1 http://www.englishheritage.org.uk/publications/midas-heritage/midas-heritage2012-v1 1.pdf (25 Mar. 2013).

Fielding, R. T., 2000. Architectural Styles and the Design of Network-based Software Architectures http://www.ics.uci.edu/ fielding/pubs/dissertation/top.htm (26 Mar. 2013).

\section{ACKNOWLEDGEMENTS}

From the Qatar Museums Authority we would like to thank H.E Shaykha al-Mayassa bint Hamad bin Khalifa Āl-Thāni (Chairperson of the Board of Trustees) and H.E. Shaykh Hasan bin Mohammed Āl-Thāni (Vice-Chairman of the Board of Trustees) for supporting and promoting this project. We would also like to express our gratitude to Ed Dolman (Chief Executive Officer), Prof. Thomas Leisten (Chief Officer of Archaeology and Conservation) and Dr. Alice Bianchi (Consultant for Archaeology and Heritage Database). We are also grateful to Prof. Vince Gaffney (University of Birmingham).

Revised June 2013 